\title{
Optimized Controller Structured to Solve Aircraft Pitch Control Problem
}

\author{
R. Monica ${ }^{1}$, R. Monica ${ }^{2}$ \\ Department of Electronics and Instrumentation Engineering, \\ St. Joseph's College of Engineering, OMR, Chennai 600119. \\ Tamilnadu, India. \\ ${ }^{1}$ rmonica.rox@gmail.com \\ 2 monica.ravichandran@yahoo.com
}

\begin{abstract}
In this paper, a comparative assessment is made based on time domain specifications and erro r criteria between various types of controllers like conventional PID, and heuristic approaches like FA, P SO and BFO based PID and I-PD structured controllers. The dynamic modelling of pitch control system i s considered on the design of an auto pilot that controls the pitch angle. To study the effectiveness of the $c$ ontrollers, PID controller, FA, PSO and BFO based PID and I-PD controllers are developed for controllin $\mathrm{g}$ the pitch angle of an aircraft. Simulation results for the response of pitch controller are presented in ste p response. Finally the performances of pitch control systems are investigated and analyzed based on com mon criteria of step's response in order to identify which control strategy delivers better performance wit $h$ respect to the desired pitch angle. The results are obtained and finally a comparative study of the error indices and time domain specifications are made between all the controllers. This experimental study conf irms that, IPD structure offers enhanced result for the reference tracking problem compared with the PI D structure. FA based PID controller has least settling time and least IAE and ISE.
\end{abstract}

Keywords-Flight pitch control, Heuristic approach, PID controller, I-PD controller, Performance eva luation.

\section{INTRODUCTION}

Present research work approves that: heuristic approach optimization is developed as a prominent practice to determine the best solutions for a class of engineering optimization issues. In the paper, number of heuristic proc edures are implemented and executed for PID and I-PD structures by the authors for a class of process models [1 ,2]. Selection of soft computing technique for a chosen problem relies on the following constrains: (i) Engineeri ng problem to be resolved, (ii) Search space dimension, (iii) Value of cost function (single or multiple), (iv) Ada ptability of the algorithm and its parameters, (v) Simplicity in execution, (vi) Optimization accuracy and (vii) Fl exibility.

In this paper, conventional controllers, such as PI and PID are tuned using the Ziegler-Nichols, Tyreus-Luyb en and relay auto tuning methods [8] and are compared with heuristic approaches like, Particle Swarm Optimiz ation (PSO), Bacterial Foraging Optimization (BFO) and Firefly Algorithm (FA) based controllers for the aircra $\mathrm{ft}$ pitch control problem. The heuristic approaches like BFO, PSO and FA are adopted to determine the optimal values of $K_{p}, K_{i}$ and $K_{d}$ for PID and I-PD structures.

The performance of the conventional and soft computing approach based controllers is assessed based on the well known time domain specifications and error criteria. Proposed work determines the best possibly tuned con troller parameters for aircraft pitch system. In this paper, the aircraft pitch control issue is addressed using the co nventional PID controllers and heuristic approaches based PID and I-PD structures.

The remaining part of the paper is as follows; section 2 outlines the description of aircraft pitch system, sectio n 3 discusses about the chosen heuristic algorithms, section 4 presents the experimental results and discussions. Finally the conclusion of the present research work is discussed in section 5.

\section{DESCRIPTION OF SYSTEM}

The movement of aircraft during flight is three dimensional and it can be represented by three principal axes-r oll, pitch and yaw .Roll is the rotation around the front to back axis. Pitch is the rotation around side to side axis. Pitch motion is the movement of the nose of the aircraft upwards and downwards. Pitch motion is responsible $f$ or the change in altitude during flight. Pitch control movement is a critical parameter during take off as well as d uring steady flight. If pitch of an aircraft is not calibrated properly then the aircraft can be stalled. Pitch moveme 
nt of aircraft is categorized under longitudinal stability and the other two parameters roll and yaw are categorize d under lateral stability. An elevator is used to control pitch and it is present on the horizontal tail surface. An el evator tilts the nose of the aircraft upwards and downwards. If the elevator rotates up, lift force on the tail decrea ses which causes the tail to lower and the nose to rise. If the elevator rotates downwards, lift force on the tail inc reases which causes the tail to rise and the nose to lower. Yaw is the rotation around the vertical axis. The move ment of aircraft is non-linear and complex to stabilize and control. Aircraft pitch varies when any of the other tw o parameters yaw or roll are varied. The forward speed of the aircraft increases when the aircraft's nose lowers a nd the forward speed decreases as the aircraft's nose rises. Hence it is required to stabilize the pitch of an aircraf $\mathrm{t}$ in order to maintain the altitude during flight. Here the authors have proposed design of conventional PI and PI D controllers using Ziegler-Nichols method, Tyreus-Luyben and relay auto-tuning methods [1] and other heuris tic approaches such as PSO, BFO and FA for stabilizing and controlling the aircraft pitch.

The mathematical equations of an aircraft are a set of six coupled non-linear differential equations. Under cert ain assumptions these equations can be decoupled and linearized using lateral and longitudinal dynamics. Longit udinal dynamics are used to govern aircraft pitch In this paper an auto pilot is designed to control the pitch of an aircraft.

Let us assume the aircraft is steady at constant altitude and velocity; hence the forces thrust, drag, weight and lift balance each other in $\mathrm{x}$ and $\mathrm{y}$ directions. .Let us assume that changing the pitch angle does not change the sp eed of an aircraft under any circumstance. Under these assumptions, the longitudinal equations are written as:

$$
\begin{aligned}
& \left(\dot{\alpha}=\mu \Omega \sigma\left[-\left(C_{L}+C_{D}\right) \alpha+1 /\left(\mu-C_{L}\right) q-\left(C_{W} \sin \gamma\right) P+C_{L}\right]\right. \\
& \dot{q}=\mu \frac{\sigma}{2 i_{y y}}\left[\left[C_{M}-\beta\left(C_{L}+C_{D}\right)\right] \alpha+\left[C_{M}+\sigma C_{M}\left(1-\mu C_{L}\right)\right] q+\left(\beta C_{W} \sin \gamma\right)\right] \\
& P \stackrel{=}{=} \Omega
\end{aligned}
$$

In this system, the input is the elevator angle $\boldsymbol{\delta}$ and pitch angle is the output of the aircraft.

Let us assume some numerical values to simplify the modeling equations:

$$
\begin{aligned}
& \dot{\alpha}=-0.313 \alpha+56.7 q=0.232 \delta \\
& \dot{q}=-0.0139 \alpha-0.426 q+0.0203 \delta \\
& \mathrm{P}^{\dot{ }}=56.7 \mathrm{q}
\end{aligned}
$$

The above values are obtained from the data of one of the Boeing's commercial aircraft.

Normally, the pitch angle of an aircraft is controlled byvarying the angle and the lift force of the rear elevator. During this situation, it is necessary to account the aerodynamic forces (lift and drug) and aircraft's inertia. As $p$ er the literature, the mathematical model of this system is a third order equation, after linearising the nonlinear $\mathrm{d}$ ynamics around an operating point.

In this paper, the well known models existing in the literature are chosen for the study.

Example 1: This model is obtained from [10,11]. This is a third order equation with a zero dead time. Eqn. (7) depicts the transfer function model and eqn. (2) shows the state-space model.

$$
\begin{aligned}
& \frac{\Delta \theta(s)}{\Delta \delta_{e}(s)}=\frac{1.151 s+0.1774}{s^{3}+0.739 s^{2}+0.921 s} \\
& {\left[\begin{array}{c}
\Delta \dot{\alpha} \\
\dot{\dot{q}} \\
\dot{\dot{\theta}}
\end{array}\right]=\left[\begin{array}{ccc}
-0.313 & 56.7 & 0 \\
-0.0139 & -0.426 & 0 \\
0 & 56.7 & 0
\end{array}\right]\left[\begin{array}{c}
\Delta \alpha \\
\Delta q \\
\Delta \theta
\end{array}\right]+\left[\begin{array}{c}
0.232 \\
0.0203 \\
0
\end{array}\right]\left[\begin{array}{ll}
\Delta \delta_{e}
\end{array}\right] y=\left[\begin{array}{lll}
0 & 0 & 1
\end{array}\right]\left[\begin{array}{c}
\Delta \alpha \\
\Delta q \\
\Delta \theta
\end{array}\right]}
\end{aligned}
$$




\section{Heuristic Algorithms AdOPTED IN THIS PAPER}

In the past decades, heuristic algorithms are emerged as a powerful tool in solving a class of process control $\mathrm{t}$ asks [1,2]. Literature evident that, even though there exists a number of heuristic methods, algorithms such as $\mathrm{Pa}$ rticle Swarm Optimization (PSO), Bacterial Foraging Optimization (BFO), and Firefly Algorithm (FA) are wide ly adapted by most of the researchers to find best possible solutions for various controller design problems [3-5].

\section{A. Particle Swarm Optimization}

PSO was discovered in the year 1995 by Kennedy and Eberhart [6] based on social activities in flock of birds and school of fish. Due to its adaptability and dominance, this technique was used to find the global optimum so lution in a complex search space during the control design problems. It is less dependent of a set of initial points than other optimization technique. It is a derivative free algorithm. The PSO algorithm has two conventional eq uations such as velocity update and position update as represented below [7-10];

$$
\begin{aligned}
& V_{i}(t+1)=W^{t} \cdot V_{i}^{t}+C_{1} R_{1}\left(P_{i}^{t}-S_{i}^{t}\right)+C_{2} R_{2}\left(G_{i}^{t}-S_{i}^{t}\right) \\
& X_{i}(t+1)=X_{i}^{t}+V_{i}(t+1)
\end{aligned}
$$

Where $W^{t}$ is inertia weight assigned as $0.75,{ }^{t}$ is the current velocity of particle, ${ }{ }_{i}(t+1)$-updated velocity of particle, $X_{i}^{t}$-current position of particle, $X_{i}(t+1)$-updated position of particle, $\mathrm{R}_{1}, \mathrm{R}_{2}$ are the random $\mathrm{n}$ umbers $[0,1]$ and $\mathrm{C}_{1}$ and $\mathrm{C}_{2}=2.1$.

\section{B. Bacterial Foraging Optimization}

BFO was also a population based approach developed by inspiring the foraging manners of E.coli bacteria [1 1]. The chief benefit of BFO algorithm is, it offers better result compared with PSO algorithm. The classical BF $\mathrm{O}$ and its variants are already chosen by the researchers to design the PID controllers for a class of systems [12,1 3]. There are two main category of BFO:

(i) Adaptive BFO (ABFO): This algorithm was proposed with the subsequent algorithm parameters: number of bacteria $(\mathrm{N})=20$, number of chemotaxis step $\left(N_{c}\right)=20$, swim length $\left(N_{s}\right)=12$, number of elimination - dispersal events $\left(N_{e d}\right)=4, N_{r e}$ (number of bacterial reproduction) $=16, P_{e d}$ (probability of bacterial elimination $/$ dispersal $)=0.25, d_{\text {attractant }}=0.1, W_{\text {attractant }}=0.2, h_{\text {repellant }}=0.1, W_{\text {repellent }}=10$, and $\lambda=20[14]$.

(ii) Enhanced BFO (EBFO): EBFO is a modified form of classical BFO algorithm. The initial algorithm parameters are assigned as follows;

Number of E.Coli bacteria $=10<N<30$ (in this work N=20); $\quad N_{c}=\frac{N}{2} ; N_{s}=N_{r e} \approx \frac{N}{3} ; N_{e d} \approx \frac{N}{4}$; $N_{r}=\frac{N}{2} ; P_{e d}=\left(\frac{N_{e d}}{N+N_{r}}\right) ; d_{\text {atractant }}=W_{\text {atractant }}=\frac{N_{s}}{N} ; \quad$ and $\quad h_{\text {repellant }}=W_{\text {repellent }}=\frac{N_{c}}{N}$.

The main advantage of EBFO compared to the classical BFO is, the number of initializing parameters to be assigned for the search in EBFO is reduced to just two i.e. N (E. Coli size) and D. Hence, in this paper , the EBFO discussed in [15] is adopted.

\section{Firefly Algorithm}

FA is one of the recent heuristic technique proposed by Yang [16]. FA is based on the duplicating the flashin $\mathrm{g}$ illumination traces produced by invertebrates such as glowworm and firefly. Normally, these insects will prod uce chemically generated light from their lower abdomen. This bio-luminescence with speckled flashing pattern s generated by glowworm/firefly is used to establish communication between two neighboring insects, to search for pray and also to find mates $[16,17]$. 
The chief parameters which decide the efficiency of the FA are the variations of light intensity and attractiven ess between neighboring fireflies.

Variation in luminance can be analytically expressed with the following Gaussian form:

$$
I(r)=I_{0} e^{-\gamma d^{2}}
$$

where $I=$ new light intensity, $I_{0}=$ original light intensity, and $\gamma=$ light absorption coefficient.

The attractiveness towards the luminance can be analytically represented as:

$$
\beta=\beta_{0} e^{-\gamma d^{2}}
$$

where $\beta=$ attractiveness coefficient, and $\beta_{0}=$ attractiveness at $\mathrm{r}=0$.

The above equation describes a characteristic distance $\Gamma=1 / \sqrt{\gamma}$ over which the attractiveness changes signi ficantly from $\beta_{0}$ to $\beta_{0} e^{-1}$. The attractiveness function $\beta(d)$ can be any monotonically decreasing functions such as the following form;

$$
\beta(d)=\beta_{0} e^{-\gamma d^{m}},(m \geq 1)
$$

For a fixed $\gamma$, the characteristic length becomes;

$$
\Gamma=\gamma^{-1 / m} \rightarrow 1, m \rightarrow \infty
$$

Conversely, for a given length scale $\Gamma$, the parameter $\gamma$ can be used as a typical initial value (that is $\gamma=1 / \Gamma \mathrm{m}$ )

The Cartesian distance between two fireflies $i$ and $j$ at $x_{i}$ and $x_{j}$, in the $n$ dimensional search space can be mat hematically expressed as;

$$
d_{i j}^{t}=\left\|X_{j}^{t}-X_{i}^{t}\right\|_{2}=\sqrt{\sum_{k=1}^{n}\left(X_{j, k}-X_{i, k}\right)^{2}}
$$

In FA, the light intensity at a particular distance $d$ from the light source $X_{i}^{t}$ obeys the inverse square la w. The light intensity of a firefly $I$, as the distance $d$ increases in terms of $I \propto 1 / d^{2}$. The movement of the at tracted firefly $i$ towards a brighter firefly $j$ can be determined by the following position update equation;

In FA, convergence speed and optimization accuracy depends mainly on the guiding parameters, which help $t$ $o$ update the agent values. Most of the preliminary heuristic algorithms are guided by the randomization operator . Due to the randomization parameter, the optimization accuracy and the convergence will not be in expected lev el in most of the search cases. Hence, in this work, Brownian distribution guided firefly algorithm is adopted to obtain enhanced values of the PID parameters [19]:

$$
X_{i}^{t+1}=X_{i}^{t}+\beta_{0} e^{-\gamma d_{i j}^{2}}\left(X_{j}^{t}-X_{i}^{t}\right)+\alpha \cdot \operatorname{sign}\left(\text { rand }^{1 / 2}\right) \oplus \text { BrownianSearch }
$$

where, $X_{i}^{t+1}=$ updated position of firefly, $X_{i}^{t}=$ initial position of firefly, and $\beta_{0} e^{-\gamma d_{i j}^{2}}\left(X_{j}^{t}-X_{i}^{t}\right)=$ attract ion between fireflies.

\section{RESULTS AND Discussions}

Here the conventional controllers PI and PID using Ziegler-Nichols, Tyreus-Luyben and relay auto-tuning me thods are designed and other heuristic approaches like Bacterial Foraging Optimization, Particle Swarm Optimiz ation and Firefly Algorithm are used to determine the optimal values of the controller. 
Table 1. Controller parameters

\begin{tabular}{|l|c|c|c|}
\hline Controller & $\mathbf{K}_{\mathbf{p}}$ & $\mathbf{K}_{\mathbf{i}}$ & $\mathbf{K}_{\mathbf{d}}$ \\
\hline ZN PI & 2.4075 & 0.1156 & - \\
\hline ZN PID & 3.2100 & 0.3082 & 8.3592 \\
\hline TL PI & 1.6700 & 0.3040 & - \\
\hline TL PID & 2.4300 & 0.4420 & 9.6500 \\
\hline RELAY PI & 0.90474 & 0.3619 & - \\
\hline RELAY PID & 1.17085 & 0.7806 & 0.4391 \\
\hline BFO PID & 0.5742 & 0.1608 & 0.1988 \\
\hline PSO PID & 0.8258 & 0.4545 & 5.0160 \\
\hline FA PID & 3.3412 & 1.1497 & 1.9859 \\
\hline BFO I-PD & 0.5742 & 0.1608 & 0.1988 \\
\hline PSO I-PD & 0.8258 & 0.4545 & 5.0160 \\
\hline FA I-PD & 3.3412 & 1.1497 & 1.9859 \\
\hline
\end{tabular}

The above table presents PI, PID I-PD structure controller values obtained through various algorithms. The $h$ euristic approaches like BFO, PSO and FA were executed multiple times and the average of obtained values are taken as the optimal controller values. The corresponding output response of these controllers tuned using vario us algorithms are shown in below graphs.

Table 2. Performance comparison of controllers tuned by various algorithms

\begin{tabular}{|c|c|c|c|c|c|}
\hline Controller & $\begin{array}{c}\text { Peak t } \\
\text { ime(s) }\end{array}$ & $\begin{array}{c}\text { Rise ti } \\
\text { me(s) }\end{array}$ & $\begin{array}{c}\text { Settling } \\
\text { time(s) }\end{array}$ & IAE & ISE \\
\hline ZN PI & 1.8 & 1.2 & 177 & 22.06 & 33.2 \\
\hline ZN PID & 1.6 & 1 & 130 & 10.6 & 13.8 \\
\hline TL PI & 2 & 1.4 & 82.8 & 18.24 & 31.16 \\
\hline TL PID & 1.6 & 1.2 & 447.4 & 11.16 & 17.27 \\
\hline RELAY PI & 7.4 & 2 & 67.6 & 16.3 & 30.96 \\
\hline RELAY PID & 11.2 & 1.4 & 47.5 & 9.48 & 17.38 \\
\hline BFO PID & 14.2 & 7.3 & 98.0 & 25.17 & 48.36 \\
\hline PSO PID & 11.6 & 5.6 & 89.1 & 7.076 & 2.958 \\
\hline FA PID & 2.2 & 1.5 & 46.2 & 3.492 & 1.693 \\
\hline BFO I-PD & 20.4 & 12.4 & 96.5 & 29.29 & 35.52 \\
\hline PSO I-PD & 13 & 7.6 & 97.1 & 32.41 & 55.19 \\
\hline FA I-PD & 13.5 & 9.4 & 58.33 & 25.94 & 40.81 \\
\hline
\end{tabular}

Performance comparison of various controllers based on time domain specifications and error criteria is prese nted in table2. It can be observed from table2. that conventional PI and PID controllers tuned by Ziegler-Nichols and Tyreus-Luyben methods have larger settling time than the PI and PID controllers tuned by relay auto-tuni ng method. PID and I-PD controllers tuned by BFO, PSO and FA have lesser settling time and lesser unwanted oscillations when compared to the conventional controllers tuned by Ziegler-Nichols and Tyreus-Luyben meth ods. I-PD structured controllers optimized by heuristic schemes like BFO, PSO and FA have larger IAE and ISE than PID controllers optimized using BFO, PSO and FA. The above table presents that PSO and FA based PID controllers have less IAE and ISE. The heuristic approach based PID and I-PD structures have relatively identic al time domain specifications but I-PD structures have larger IAE and ISE than BFO, PSO and FA based PID co ntroller. 


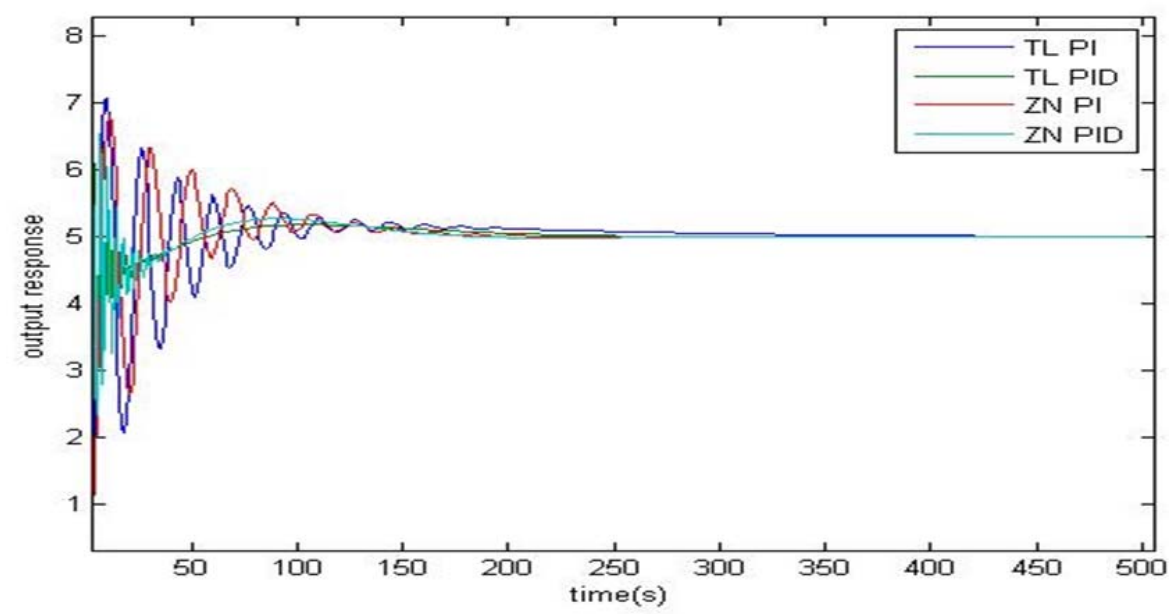

Figure 1. Comparison of PI and PID tuned by Z-N and Tyreus-Luyben methods

From Fig 1, it can be observed that conventional PI and PID controllers have enormous unwanted oscillation s.

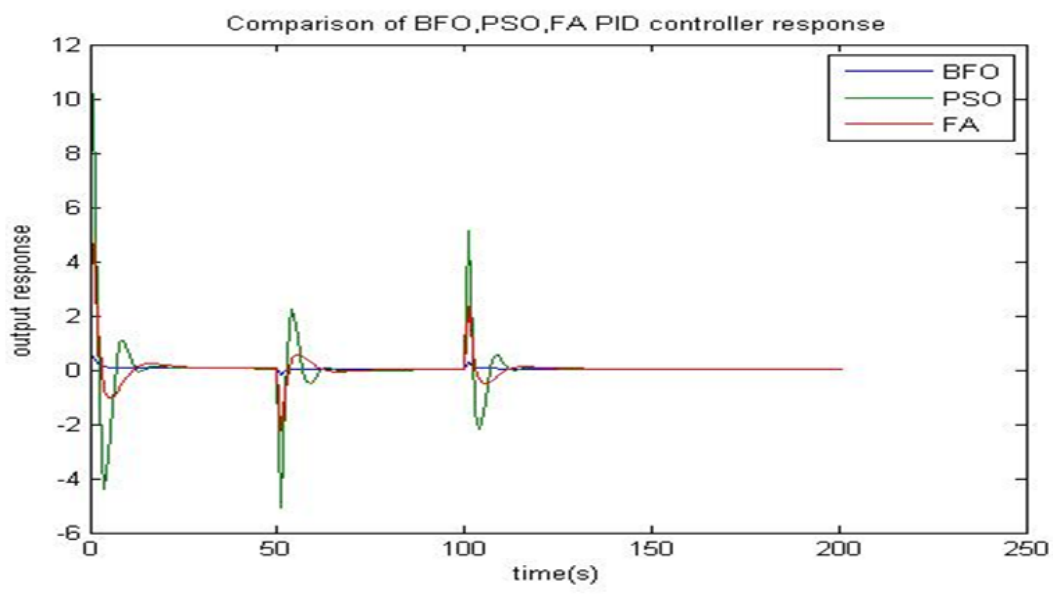

Figure. 2(a). Comparison of BFO, PSO and FA PID controller response

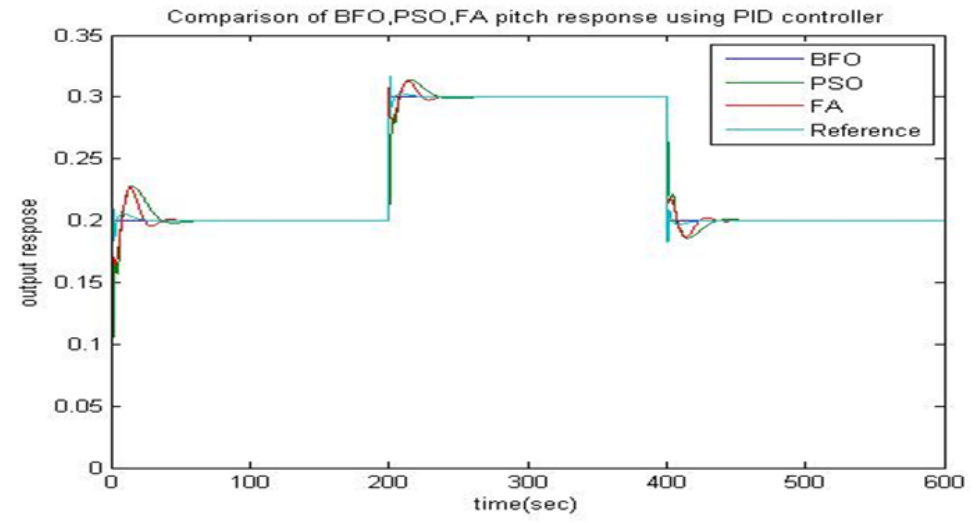

Figure 2(b) Comparison of BFO, PSO and FA plant response with PID controller 


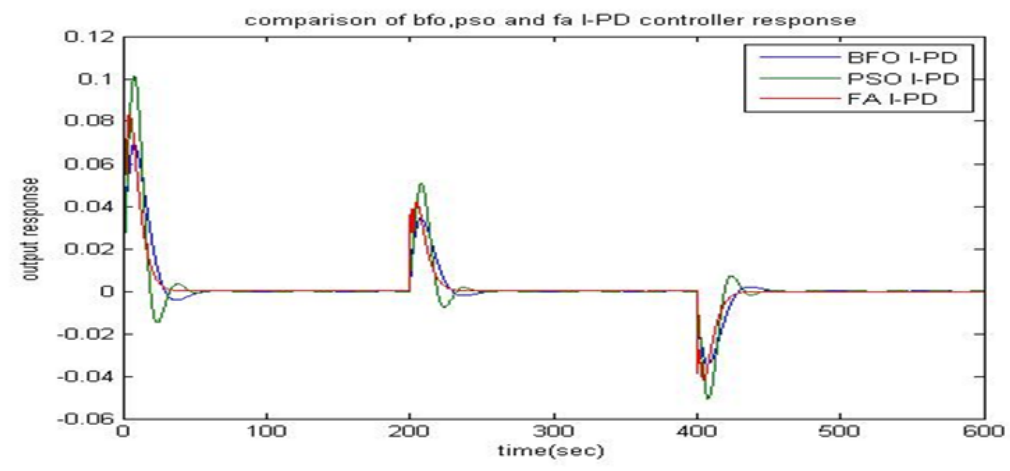

Figure. 3(a). Comparison of BFO, PSO and FA I-PD controller response

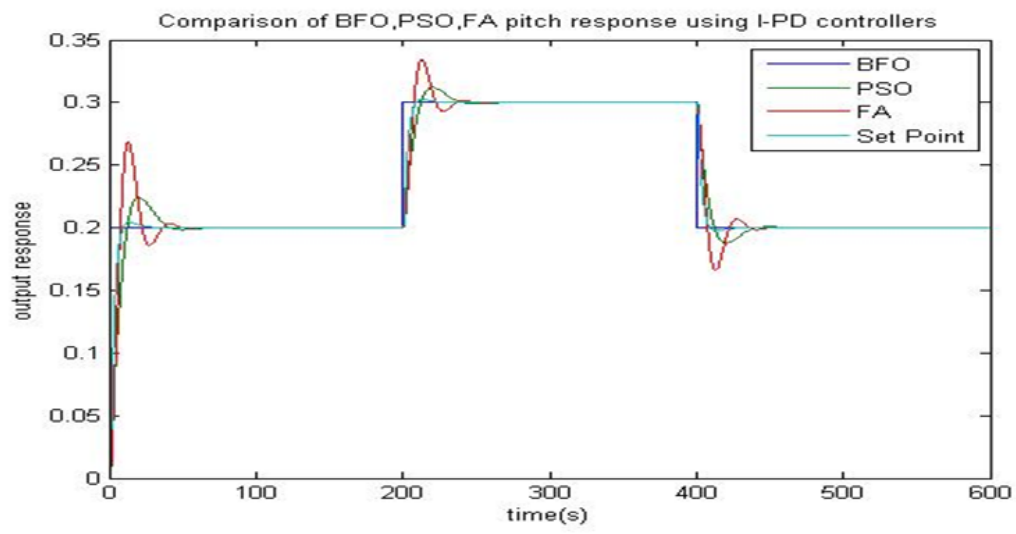

Figure. 3(b) Comparison of BFO, PSO and FA plant response with I-PD controller

From Fig. 2(a) and 2(b) it is observed that PID controller values obtained by heuristic approaches have lesser oscillations then conventional PID controller. I-PD structure reduces the unwanted oscillations but has larger IA E and ISE than PID controllers. From Fig. 3(a) and 3(b) it is observed that BFO, PSO and FA based PID control lers have relatively smaller peak overshoot than BFO, PSO and FA based I-PD structured controllers.

From table2, it is observed that PID controller values obtained by Firefly Algorithm has less peak time, rise ti me, settling time and also less IAE and ISE. PID controller optimized by FA has best output response with less unwanted oscillations, least settling time and least error indices IAE and ISE.

\section{CONCLUSION}

This experimental work proposes heuristic approaches to design the PID and I-PD controllers for the aircraft pitch control. In this work, the heuristic approaches, such as the BFO, PSO and FA are considered and their perf ormances are evaluated based on the time domain values and the error values. From this study, we observe that, the reference tracking performance offered by the chosen algorithms are nearly identical. The BFO, PSO and F A based I-PD structures provide better response by reducing the unwanted oscillations. FA based PID controller has best output response with least settling time and minimum error indices IAE and ISE.

\section{REFERENCES}

[1] B. Wayne Bequette, "Process Control - Modeling, Design and Simulation," Prentice - Hall of India Pvt Ltd, 2003.

[2] R. PadmaSree, and M. Chidambaram, "Control of Unstable Systems," Narosa Publishing House, India, 2006.

[3] K. Suresh Manic, V. Rajinikanth, SarathAnanthasivam, Uma Suresh, "Design of Controller in Double Feedback Control Loop-An Analysis with Heuristic Algorithms," Chemical Product and Process Modeling, vol.10, no.4, pp. 253-262, 2015.

[4] V. Rajinikanth, and K. Latha, "Optimization of PID controller parameters for unstable chemical systems using soft computing technique," International Review of Chemical Engineering, vol.3, no.3, pp. 350-358, 2011.

[5] V. Rajinikanth, and K. Latha, "Setpoint weighted PID controller tuning for unstable system using heuristic algorithm," Archives of Control Sciences, vol.22, no.4, pp. 481-505, 2012.

[6] J. Kennedy, and R. Eberhart, "Particle Swarm Optimization," in proceedings of IEEE International Conference on Neural, pp. 1942$1948,1995$. 
[7] V. Rajinikanth, and K. Latha, "Tuning and Retuning of PID Controller for Unstable Systems Using Evolutionary Algorithm," ISRN Chemical Engineering, vol. 2012, Article ID 693545, 11 pages, 2012.

[8] Wael. M. Korani, Hassen Taher Dorrah, and Hassan M. Emara, "Bacterial foraging oriented by particle swarm optimization strategy for PID tuning," Proceedings of the $8^{\text {th }}$ IEEE international conference on Computational intelligence in robotics and automation, pp. 445$450,2008$.

[9] K. Latha, V. Rajinikanth, and P. M. Surekha, "PSO-Based PID Controller Design for a Class of Stable and Unstable Systems," ISRN Artificial Intelligence, vol. 2013, Article ID 543607, 11 pages, 2013.

[10] N. Wahid, N. Hassan, M.F. Rahmat, and S. Mansor, "Application of intelligent controller in feedback control loop for aircraft pitch control," Australian Journal of Basic and Applied Sciences, vol.5, No.12, pp.1065-1074, 2011.

[11]M. Zugaj, and J.J. Narkiewicz, "Autopilot supported by nonlinear model following reconfigurable flight control system", Journal of Aerospace Engineering, Vol. 23, 2010, No. 4, pp. 339-347.

[12] V. Rajinikanth, and K. Latha, "Bacterial foraging optimization algorithm based PID controller tuning for time delayed unstable system," The Mediterranean Journal of Measurement and Control, vol.7, no.1, pp. 197-203, 2011.

[13] V. Rajinikanth, and K. Latha, "PID controller tuning for magnetic suspension system using evolutionary algorithm,"International Review of Mechanical Engineering, vol.6, no.5, pp. 988-995 2012.

[14] V. Rajinikanth, and K. Latha, "I-PD controller tuning for unstable system using bacterial foraging algorithm: a study based on various error criterion," Applied Computational Intelligence and Soft Computing, vol. 2012, Article ID 329389, 10 pages, 2012.

[15]V. Rajinikanth, and K. Latha, "Controller Parameter Optimization for Nonlinear Systems Using Enhanced Bacteria Foraging Algorithm," Applied Computational Intelligence and Soft Computing, vol. 2012, Article ID 214264, 12 pages, 2012.

[16]X.-S. Yang, "Firefly algorithms formultimodal optimization," in Stochastic Algorithms: Foundations and Applications, Lecture Notes in Computer Science, vol. 5792, pp. 169-178, 2009.

[17]N. S.M. Raja, V. Rajinikanth, and K. Latha, "Otsu Based Optimal Multilevel Image Thresholding Using Firefly Algorithm,” Modelling and Simulation in Engineering, vol. 2014, Article ID 794574, 17 pages, 2014

[18] V. Rajinikanth, and M.S. Couceiro, "RGB histogram based color image segmentation using firefly algorithm," Procedia Computer Science, vol.46, pp.1449-1457, 2015.

[19]R. Sivakumar, V. Rajinikanth, and D. Sankaran, "Multi-loop PI controller design for TITO system: an analysis with BA, FA, PSO and BFO," Australian Journal of Basic Applications Sciences, vol.9, no.16, pp.249-54, 2015. 